\title{
Total-Electron-Yield Measurements by Soft X-Ray Irradiation of Insulating Organic Films on Conductive Substrates
}

\author{
Yasuji Muramatsu*t and Eric M. GulliKson** \\ *Graduate School of Engineering, University of Hyogo, 2167 Shosha, Himeji, Hyogo 671-2201, Japan \\ **Center for X-Ray Optics, Lawrence Berkeley National Laboratory, 1 Cyclotron Road, Berkeley, CA 94720, \\ USA
}

\begin{abstract}
The photocurrent (sample current) of insulating $0.7-\mu \mathrm{m}$ thick polyethylene terephthalate (PET) films on conductive substrates $(\mathrm{C}, \mathrm{Au}, \mathrm{Cu})$ was clearly measured through the substrates during soft X-ray irradiation on the PET films. X-ray absorption measurements of the PET/conductive-substrates using the total-electron-yield (TEY) method by measuring sample current easily provide the X-ray absorption spectra (XAS) of PET films, which are independent of the substrates. From additional X-ray absorption measurements using self-standing PET/Au and Au/PET-films, $I-V$ measurements, and thickness-dependent sample current measurements, it can be confirmed that electrically conductive paths form in the insulating PET film in thickness direction along the soft X-ray beam trajectory. Such phenomena enable easy and simple TEY-XAS measurements of insulating $\mu \mathrm{m}$-order-thick samples.
\end{abstract}

Keywords Soft X-ray, X-ray absorption, photocurrent, total electron yield, insulating

(Received May 19, 2020; Accepted August 13, 2020; Advance Publication Released Online by J-STAGE August 21, 2020)

\section{Introduction}

The yield of electrons (e.g., photoelectrons, Auger electrons, and secondary electrons) emitted from matter by X-ray irradiation is generally proportional to the X-ray absorption intensity. Thus, the photocurrent can flow in electrically conductive matter by X-ray irradiation when it is grounded. The photocurrent measured in grounded matter is usually called the sample current. Monitoring the sample current during X-ray energy scanning is easy using X-ray absorption spectroscopy (XAS), which is a total electron yield (TEY) method. ${ }^{1,2}$ In particular, the TEY method is commonly used in soft X-ray absorption spectroscopy because the fundamental transmission method for XAS is difficult to apply to soft X-ray absorption measurements.

Although numerous XAS studies have used the TEY method to examine electrically conductive materials, it is difficult to apply to insulating bulk materials because sample charge-ups distort the TEY. To avoid or suppress charge-up effects of insulating bulk samples during X-ray irradiation, bulk samples are often powdered, and the powdered samples are placed on conductive substrates. ${ }^{3,4}$ The TEY of the insulating powder samples can be easily measured via the sample surface in contact with a conductive substrate. This system effectively monitors the surface sample current of insulating particles, but it requires deconstructive preparations of bulk samples.

Recently, photoemission electron microscopy (PEEM) measurements of insulating materials have been performed by coating $\mathrm{Au}$ thin films near the observation area. ${ }^{5}$ The Au film

$\dagger$ To whom correspondence should be addressed.

E-mail: murama@eng.u-hyogo.ac.jp works to release the positive charge created by a photoemission. A similar approach to detect the sample current in insulating bulk samples has also been proposed in TEY-XAS. ${ }^{6}$ In this approach, an electrode is placed on the sample surface and then $\mathrm{X}$-ray beams irradiate the sample near the electrode. Such a surface-current-monitoring method can obtain the XAS of insulating bulk samples without destructive sample preparation.

We recently found another method to easily observe the TEY of insulating bulk samples. In our method, insulating film samples are placed on conductive substrates, and the sample current generated by soft X-ray irradiation can be efficiently detected through the backside of the substrate. This paper demonstrates TEY measurements of insulating organic films placed on conductive substrates and experimentally confirms electrical conduction through the insulating films by soft X-ray irradiation.

\section{Experimental}

The main sample was a $0.7-\mu \mathrm{m}$ thick polyethylene terephthalate (PET) film $\left(\mathrm{C}_{10} \mathrm{H}_{8} \mathrm{O}_{4}\right)_{\mathrm{n}}$, supplied by Rigaku Corporation. The PET film can be used for the window material of X-ray detectors. Commercially available 2 and $4.5-\mu \mathrm{m}$ thick PET films, which were supplied by Teijin Ltd and Toray Industries Inc., respectively, were also used for the thickness-dependent measurements. Prior to the XAS measurements, the measured electric resistivity of the PET film was larger than $10^{12} \Omega \mathrm{cm}$, confirming that the PET film is an insulator.

Soft X-ray absorption measurements were performed at beamline BL-6.3.27 in the Advanced Light Source (ALS) using synchrotron radiation (SR). SR beams in the soft X-ray region were monochromatized by a $1200 \mathrm{~mm}^{-1}$ grating with a $40-\mu \mathrm{m}$ slit. 
(a) Self-standing PET for Trans-XAS

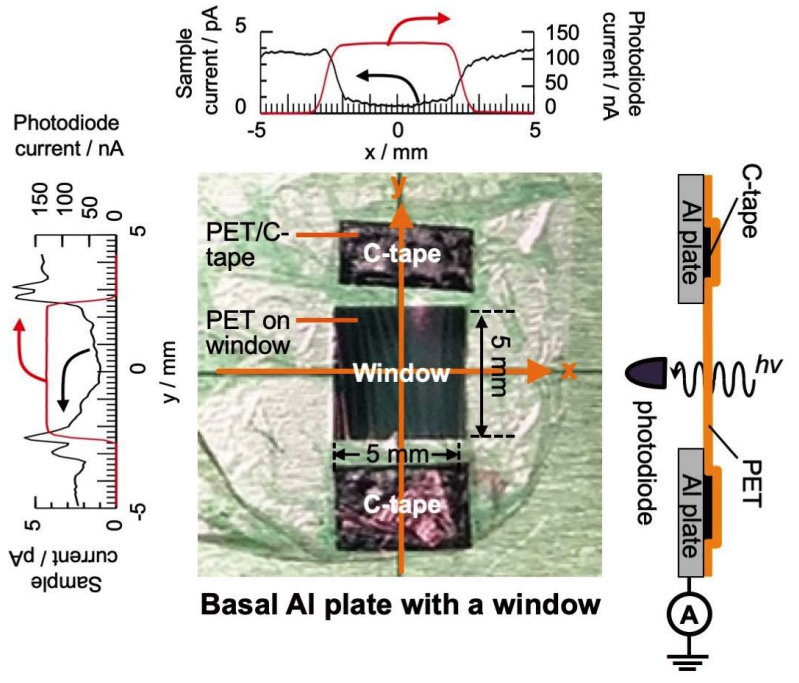

(b) PET on conducting substrates for TEY-XAS

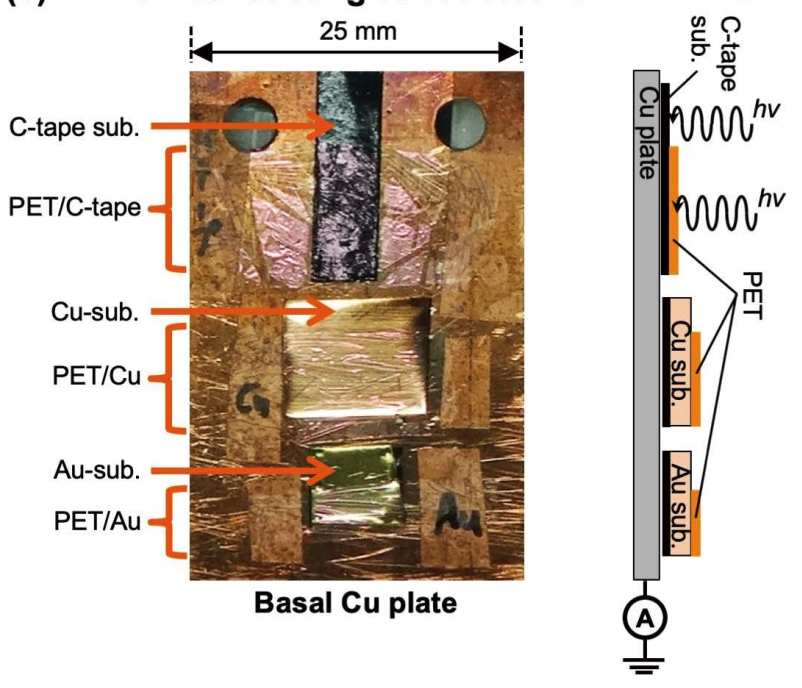

Fig. 1 Photos and cross-sectional schematic drawings of the sample setup for X-ray absorption measurements using the Trans-XAS mode for self-standing PET films (a) and TEY-XAS mode using PET films on conductive substrates (b). Line-scanning spectra along $x$ - and $y$-directions of sample current and transmitted X-ray intensity measured with a photodiode are displayed in (a).

XAS was measured in the $\mathrm{C} K(260-310 \mathrm{eV}), \mathrm{O} K(510-$ $560 \mathrm{eV}$ ), and $200-600 \mathrm{eV}$ regions without filters. In BL-6.3.2, various filters are equipped to suppress higher-order beams: $\mathrm{Ti}$ filter for the $\mathrm{C} K$ region and $\mathrm{Cr}$ filter for the $\mathrm{O} K$ region. From previous measurements, ${ }^{8}$ it has been clarified that slightly higher-orders can be observed in the $\mathrm{O} K$ region without a $\mathrm{Cr}$ filter, while higher-orders are involved in the $\mathrm{C} K$ regions without a $\mathrm{Ti}$ filter. Additionally, the calculated attenuation lengths of soft X-rays for PET are $0.6 \mu \mathrm{m}$ in $\mathrm{O} K$ and $0.2 \mu \mathrm{m}$ in the $\mathrm{C} K$ region. Hence, we should consider the attenuation lengths when discussing the $\mathrm{C} K$ - and $\mathrm{O} K$-XANES profiles. The incident SR beam size was measured as $25 \mu \mathrm{m}^{\mathrm{V}} \times 150 \mu \mathrm{m}^{\mathrm{H}}$ by a knife-edge method. All measurements were achieved under the vacuum of $10^{-5} \mathrm{~Pa}$.

For the transmission mode (denoted by Trans-XAS), the transmitted X-ray intensity was monitored with a photodiode (PD) located downstream of the PET film. The X-ray transmittance $\left(I / I_{0}\right)$ was obtained from the photodiode currents through the PET film $(I)$ and direct SR beam $\left(I_{0}\right)$. The absorbance was determined as $-\ln \left(I / I_{0}\right)$. For the TEY mode (TEY-XAS), the spectral intensity $\left(I s_{0}\right)$ of the incident soft $\mathrm{X}$-rays was monitored by the sample current of a clean Au plate after washing with sodium hypochlorite. ${ }^{9}$ For each sample, the TEY was obtained from the sample current $(I s)$ divided by $I s_{0}$ of the Au plate. That is, TEY $=I s / I s_{0}$.

Figure 1 shows photos and cross-sectional schematic drawings of the sample setup for the XAS measurements using the transmission and TEY modes. For the Trans-XAS mode shown in Fig. 1(a), the self-standing PET film was placed on a window $\left(5 \mathrm{~mm}^{\mathrm{V}} \times 5 \mathrm{~mm}^{\mathrm{H}}\right)$ of a basal aluminum $(\mathrm{Al})$ plate with a conductive C-tape. SR beams normally irradiate the center of the PET film on the window. Soft X-rays transmitted through the PET film were detected by a photodiode detector. The Al plate was connected to a galvanometer. Thus, the TEY of a selfstanding PET film can be simultaneously measured by monitoring the surface sample current from the backside of the PET film center through the $\mathrm{C}$-tape and $\mathrm{Al}$ plate. Line-scanning spectra along $x$ - and $y$-directions of the sample current and transmitted X-ray intensity measured with a photodiode are displayed in Fig. 1(a). The line-scanning profiles show that the sample current can be clearly detected on the PET/C-tape ( $y$-scanning profile) and PET/Al ( $x$-scanning profile) positions.

For the TEY-XAS mode, PET films partially covered the conductive C-tape, $\mathrm{Cu}$ plate, and Au plate substrates (Fig. 1(b)), which are denoted by PET/C, PET/Cu, and PET/Au, respectively. The PET/conductive-substrate $(\mathrm{C}, \mathrm{Cu}, \mathrm{Au})$ samples were placed on a basal $\mathrm{Cu}$ plate with $\mathrm{C}$-tape. SR beams normally irradiate at the center of the PET/conductive-substrates or the conductive substrates. The sample current induced by soft X-ray irradiation was measured through the basal $\mathrm{Cu}$ plate by a galvanometer. Hence, if the sample current of PET is detected on PET/ conductive-substrates, the current flows in the thicknessdirection of the PET films but not on the surface.

Other experiments were also performed to confirm the flow of sample current inside PET films. The sample setup for these experiments is described later.

\section{Results and Discussion}

\section{Absorbance spectra and TEY spectra}

Figure 2(a) shows the spectra in the $200-600 \mathrm{eV}$ region of the self-standing PET film. The transmittance and absorbance spectra were obtained from the photodiode currents of the direct beam $\left(I_{0}\right)$ and the transmitted beam $(I)$ through the film. The photodiode current for the transmitted beam is several tens of $\mathrm{nA}$ to $400 \mathrm{nA}$, corresponding to a transmittance $\left(I / I_{0}\right)$ of $0.2-0.6$. This shows that SR beams can sufficiently transmit in PET. The absorption peaks at the $\mathrm{C} K$ and $\mathrm{O} K$ edges in the absorbance $\left(-\ln \left(I / I_{0}\right)\right)$ spectrum show the carbon and oxygen components in PET. On the other hand, a weak sample current $(I s)$ is detected in the $\mathrm{C} K-\mathrm{O} K$ region through the self-standing film. As mentioned in the previous section, the detected sample current is the surface current on PET. Hence, monitoring the surface current, TEY surf $_{\text {-XAS }}\left(I s / I s_{0}\right)$, provides the very weak absorption structure.

Figure 2(b) shows the sample current and TEY-XAS spectra in the $200-600 \mathrm{eV}$ region of the PET/conductive-substrates $(\mathrm{Au}, \mathrm{C}, \mathrm{Cu})$ and the individual conductive substrates. The sample currents $(I s)$ of the conductive substrates of $\mathrm{Au}\left(I s_{0}\right)$, $\mathrm{C}$-tape, and $\mathrm{Cu}$ are several tens of pA. Additionally, those of $\mathrm{PET} /$ conductive-substrates were also monitored and compared 


\section{(a) Self-standing PET}

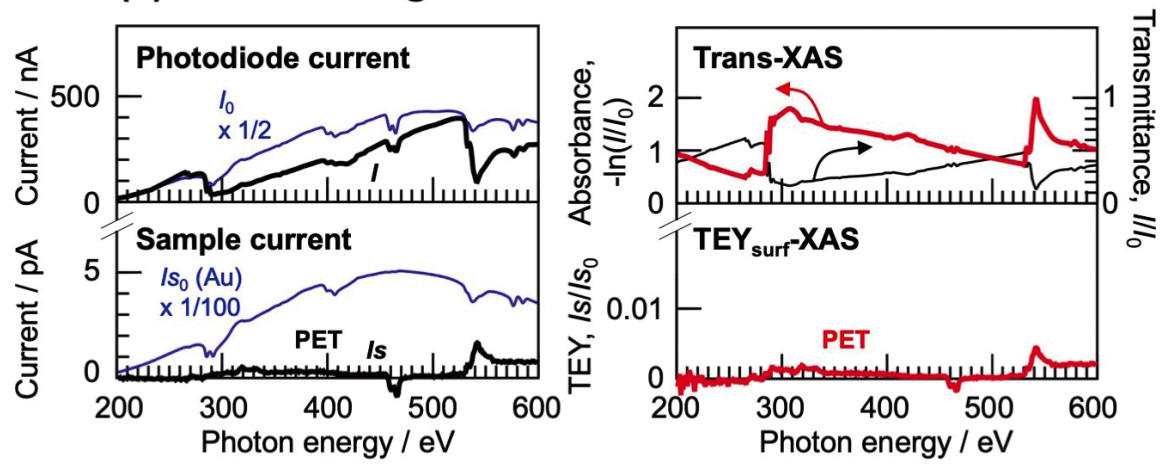

(b) PET/conducting-substrates
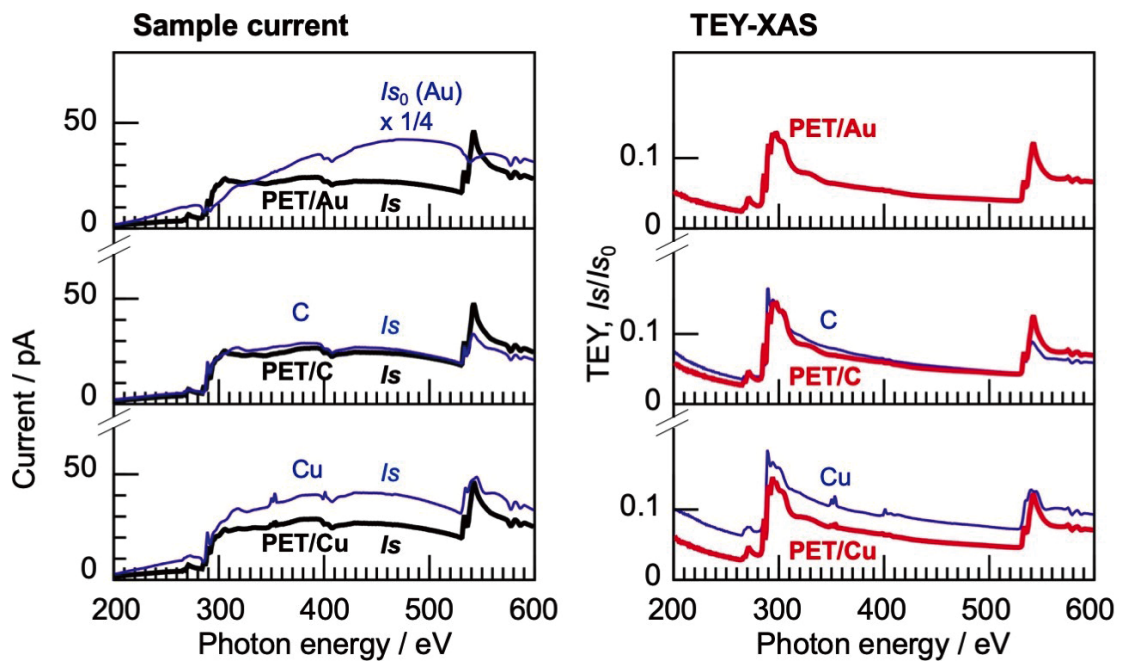

Fig. 2 Upper panels (a) show the spectra in the $200-600 \mathrm{eV}$ region of the self-standing PET film. Photodiode current and sample surface current spectra (left). Transmittance and absorbance spectra with a Trans-XAS mode and the TEY surf $\mathrm{XAS}$ (right). Lower panels (b) show the spectra in the $200-600 \mathrm{eV}$ region of the PET/conductive-substrates. Sample current spectra (left) and TEY-XAS (right).
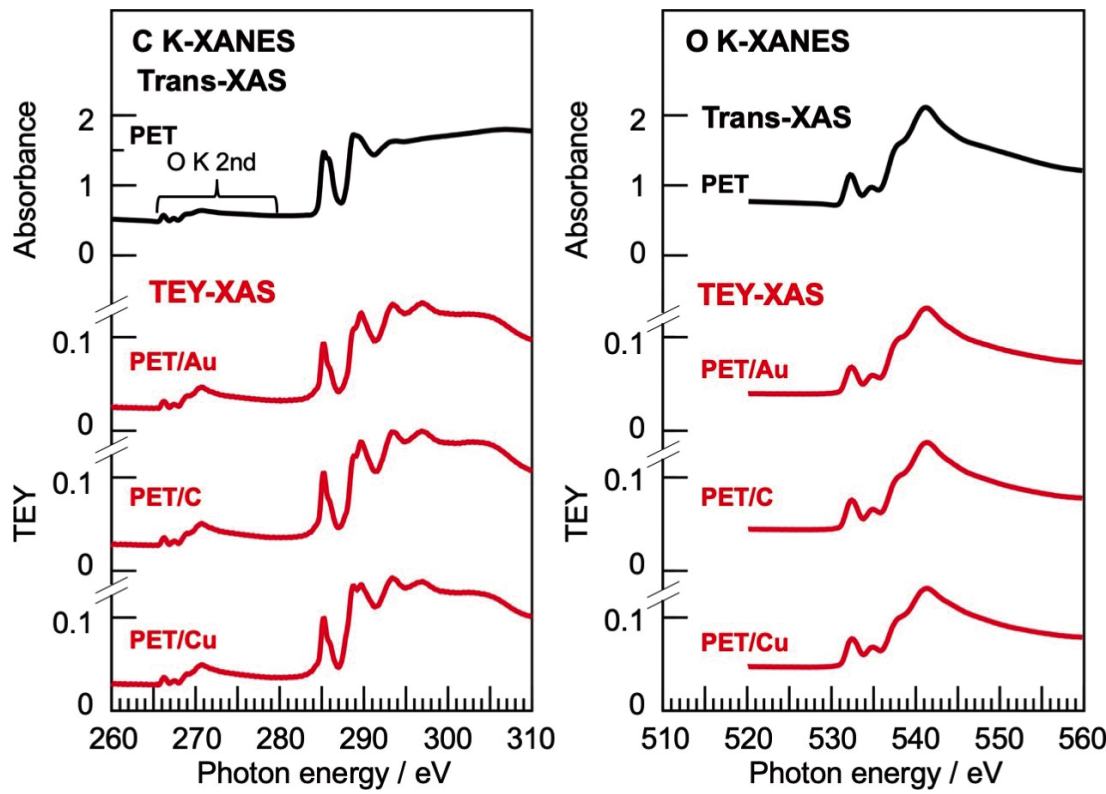

Fig. 3 XANES in the $\mathrm{C} K$ and $\mathrm{O} K$ regions of PET measured with the Trans-XAS mode (absorbance spectra) and those of PET/conductive-substrates with the TEY-XAS mode. 


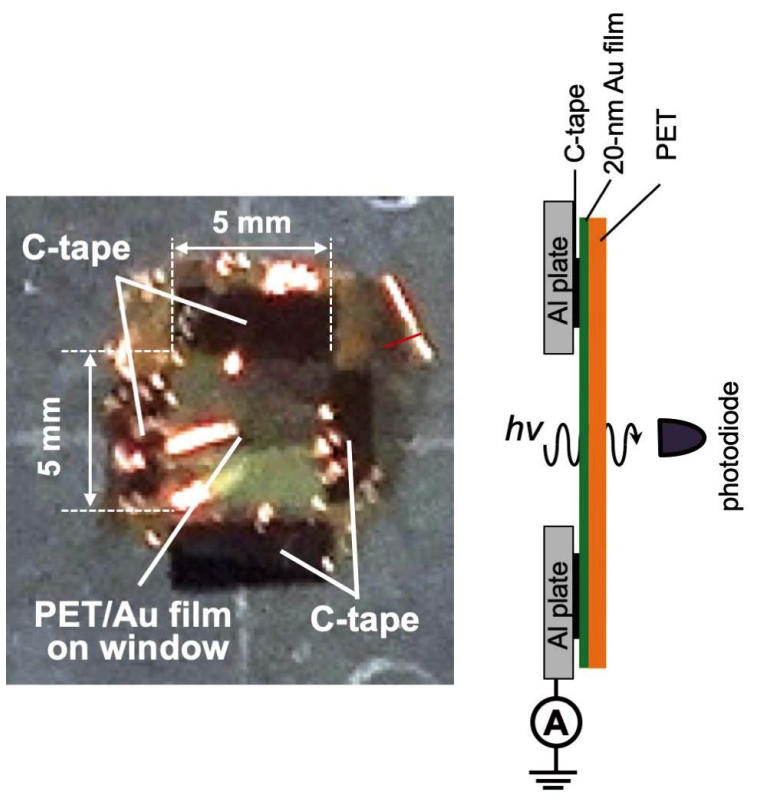

(a) Au/PET

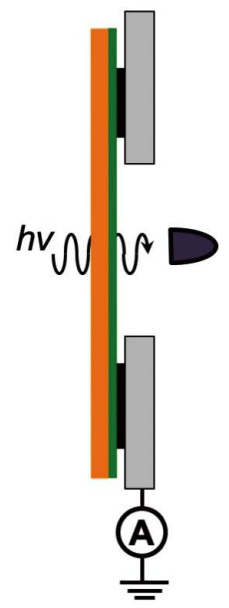

(b) PET/Au

Fig. 4 Photo and cross-sectional schematic drawings of Au/PET film (a) and PET/Au film (b) configurations.

to the substrates. The TEY-XAS $\left(I s / I s_{0}\right)$ of each PET/conductivesubstrate exhibits the same profile, which differs from that of the substrates. This suggests that the TEY-XAS of PET/ conductive-substrates originate from PET films and are independent of the substrate.

Figure 3 shows the XANES in the $\mathrm{C} K$ and $\mathrm{O} K$ regions of PET measured in the Trans-XAS mode (absorbance spectra) and those of PET/conductive-substrates in the TEY-XAS mode. In the C $K$-XANES, each TEY-XAS of the PET/conductivesubstrate exhibits the same profile, which is similar to the absorbance spectrum measured in the Trans-XAS mode. The slight difference between the TEY-XAS and Trans-XAS modes can be explained by the thickness of the PET film. The $0.7-\mu \mathrm{m}$ thick PET film is too thick for transmittance measurements in the $\mathrm{C} K$ region with a strong $\mathrm{X}$-ray absorption. Hence, the profile of the absorbance spectrum shows a broader and saturated profile at the $C K$ edge compared to that of TEY-XAS. In the O $K$-XANES, each TEY-XAS of the PET/conductivesubstrates and the absorption spectrum in the Trans-XAS mode exhibit the same profiles. The same $\mathrm{O} K$-XANES profiles in the TEY-XAS of PET/conductive-substrates and in the Trans-XAS of the self-standing PET film mean that the sample current observed in the PET/conductive-substrates comes from the PET film and is independent of the substrate. It also suggests that the sample current flows through the PET films in the thickness direction but not on the film surface.

\section{Measurements of sample current flow inside the PET film}

To confirm the electric conduction path formed inside the PET films by soft X-ray irradiation, additional experiments were performed. Figure 4 shows a photo and cross-sectional schematic drawing of the sample. Au films (20-nm thick) were deposited on $0.7-\mu \mathrm{m}$-thick PET films. The Au/PET film was placed on the window of the basal Al plate described above. The Au-film side was attached on the Al plate with C-tape. Hence, the sample current formed in the film by soft X-ray irradiation was detected through the Au-film. Two configurations in the same sample were used for the XAS measurements: a Au/ PET configuration where the SR beams irradiated onto the Aufilm side (Fig. 4(a)) and a PET/Au configuration where the SR beams irradiated onto the PET side (Fig. 4(b)). The transmitted soft X-rays were also simultaneously detected by a photodiode during the sample current measurements through the $\mathrm{C}$-tape and Al plate by a galvanometer.

Figure 5 shows the sample current and TEY-XAS spectra in the $\mathrm{C} K$ region of the Au/PET film (a) and the PET/Au film (b) configurations. In the Au/PET, the spectral profile of the sample current $(I s)$ is the same as that of Au plate $\left(I s_{0}\right)$. Hence, TEYXAS $\left(I s / I s_{0}\right)$ exhibits no absorption structure. This is reasonable because many electrons are emitted from the Au surface of the $\mathrm{Au} / \mathrm{PET}$ film by soft X-ray irradiation and the sample current spectrum reflects the $\mathrm{Au}$ in the Au/PET film. On the other hand, in PET/Au, the sample current $(I s)$ spectrum differs from $I s_{0}$ and the TEY-XAS $\left(I s / I s_{0}\right)$ drops over the $285-\mathrm{eV}$ region. This TEYXAS profile resembles the transmittance spectrum measured in the Trans-XAS mode. The absorbance spectrum of the PET/Au film is almost the same as that of the self-standing PET film shown in Fig. 3. This means that SR beams sufficiently transmit the PET/Au film and that electrons should not only be emitted from the PET side by the incident SR beams but also from the backside $\mathrm{Au}$ by the transmitted SR beams. Considering the much larger electron yield from Au than PET, the sample current spectrum of the PET/Au film should include the sample current of the backside Au. Therefore, to obtain the TEY-XAS of $\mathrm{PET} / \mathrm{Au}, I s_{0}$ should be corrected as $I s_{0} \times T$, where $T$ is the transmittance. The TEY-XAS obtained by $I s /\left(I s_{0} \times T\right)$ exhibits the same $\mathrm{C} K$-XANES profile as the PET/conductive-substrates shown in Fig. 3. This means that the sample current of the PET surface induced by soft X-ray irradiation flows inside the PET film and can be detected through the backside Au.

Sample current signal can be detected to compensate for emitted electrons from surface to several-nm depth, because the mean free path of electrons excited by soft X-rays in solid is several $\mathrm{nm}$. However, photoelectrons by soft X-ray irradiation 

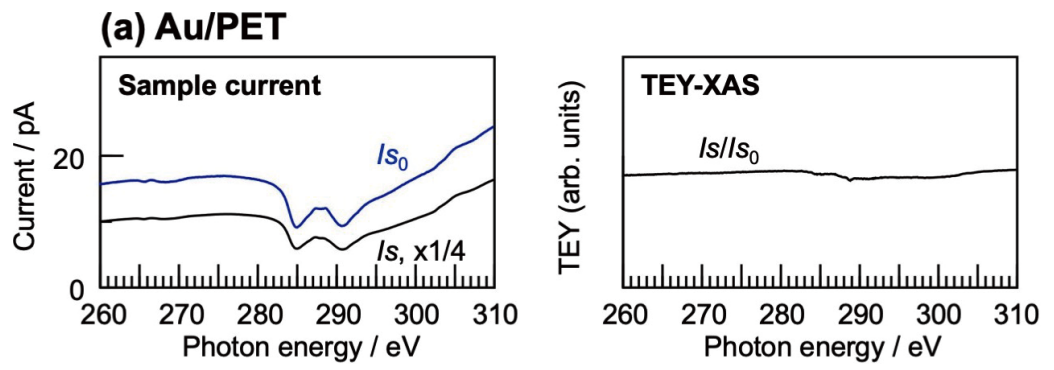

\section{(b) PET/Au}
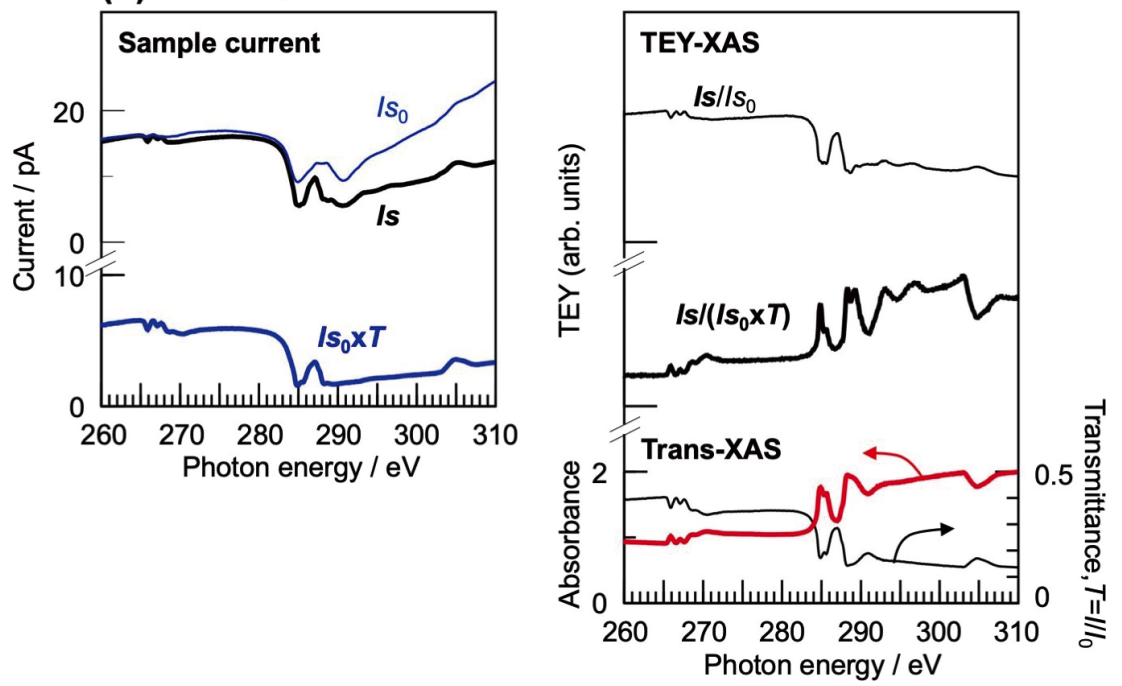

Fig. 5 Spectra in the $C K$ region of the sample current and TEY-XAS in Au/PET (a) and in PET/Au (b). Transmittance and absorption spectra measured with the Trans-XAS mode of the Au/PET and $\mathrm{PET} / \mathrm{Au}$ are also displayed.

should be generated not only on the PET surface but also inside the PET film along the soft X-ray beam trajectory. Therefore, electron-hole pairs should form along the soft X-ray beam inside the PET film. If the electron-hole pairs along the soft $\mathrm{X}$-ray trajectory provide an electric conduction path and soft $\mathrm{X}$-rays are sufficiently transmitted in the film, then the sample current of the PET film surface will be detected at the backside of the film through the inside conduction path. SR beams can sufficiently transmit through a $0.7-\mu$ m-thick PET film, as shown in Figs. 2(a) and 5(b). Hence, the conduction path along the soft $\mathrm{X}$-ray beam trajectory would be formed inside the PET film.

\section{I-V Measurements of the PET film during soft X-ray irradiation}

To further confirm the flow of sample current in the thickness direction of the PET film during soft X-ray irradiation, the relationship between sample current $(I)$ and voltage $(V)$ in the PET film was measured. Figure 6 shows a photo and a crosssectional schematic drawing of the sample setup for the $I-V$ measurements. The PET film was placed between conductive $\mathrm{C}$-tapes (front and back) on the acrylic plate. The C-tape (front) masked with a polytetrafluoroethylene (PTFE) sheet has a window $\left(2 \mathrm{~mm}^{\mathrm{V}} \times 2 \mathrm{~mm}^{\mathrm{H}}\right)$. SR beams with the size of $25 \mu \mathrm{m}^{\mathrm{V}} \times 150 \mu \mathrm{m}^{\mathrm{H}}$ irradiated the PET/C-tape (back) through the window. The sample current of the PET/C-tape (back) detected through the C-tape (back) was automatically measured by a galvanometer during SR-beam scanning. The voltage of the PET film detected between the C-tapes (front and back) was manually measured by using a conventional digital multimeter (Fluke 77) at four energy positions near $\mathrm{C} K$ and $\mathrm{O} K$ edges by
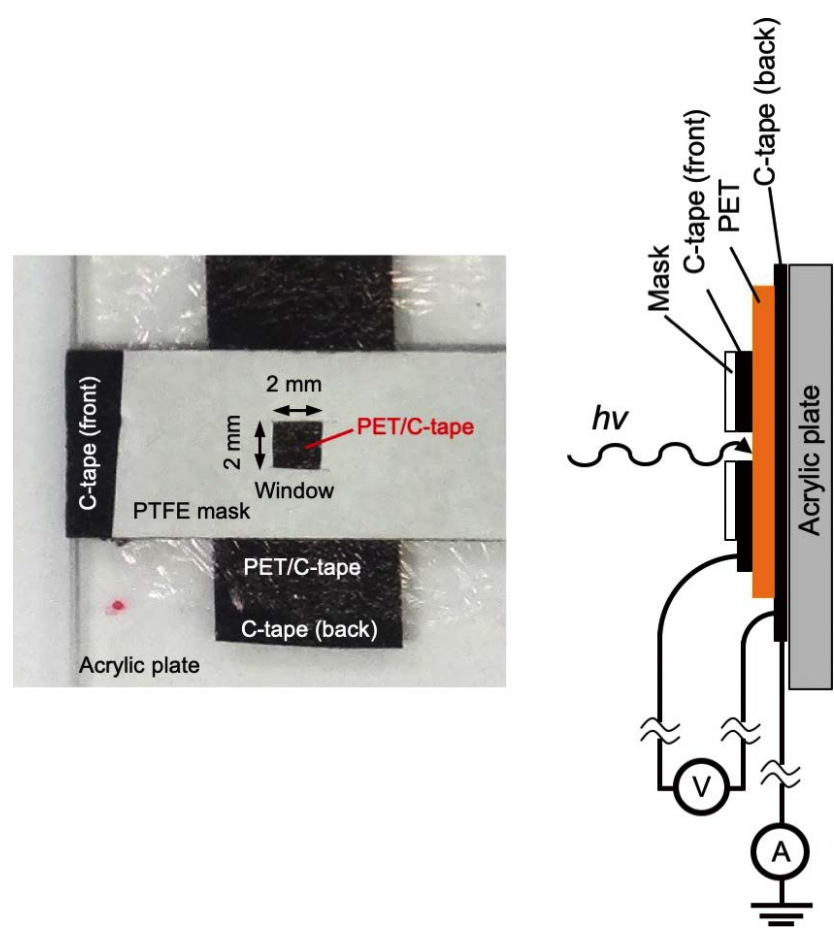

Fig. 6 Photo and cross-sectional schematic drawing of the PET/Au film sample for $I-V$ measurements. 

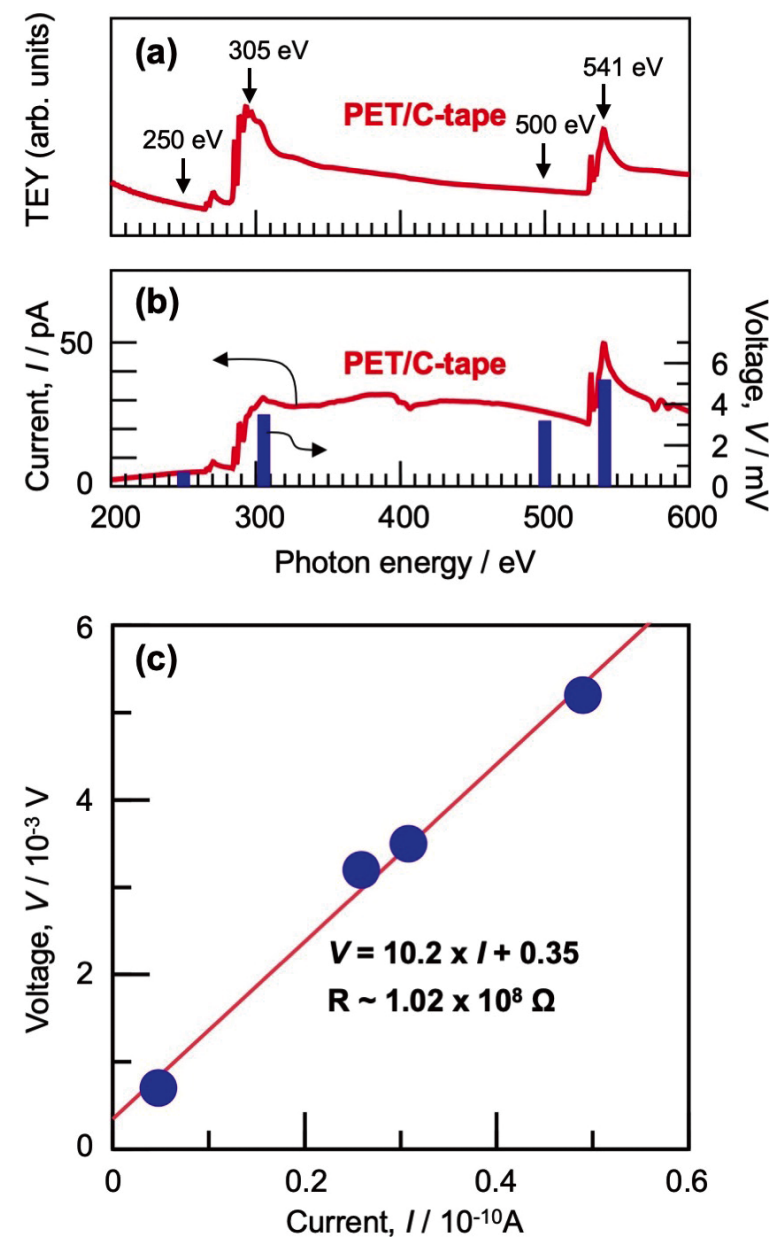

Fig. 7 Upper panels show the TEY spectrum (a) and the sample current spectrum with voltages near the $\mathrm{C} K$ and $\mathrm{O} K$ edges (b) of PET/C-tape sample. Lower (c) shows the $I-V$ plot measured near the $\mathrm{C} K$ and $\mathrm{O} K$ edges.

stopping the SR-beam scanning.

Figure 7(a) shows the TEY spectrum in the $200-600 \mathrm{eV}$ region of the PET/C-tape (back), which is the same as the TEYspectra shown in Fig. 2(b). On the spectrum, energy positions for $V$ measurements are shown by arrows at 250, 305 (on the $\mathrm{C}$ $K$ edge), 500, and 541 (on the $\mathrm{O} K$ edge) eV. Figure 7(b) shows the sample current $(I)$ spectrum and voltage $(V)$ at the above four energy positions. Figure 7 (c) shows the $I-V$ plot measured at the four energy positions. The proportional $I-V$ profiles mean that the sample current induced by the soft X-ray irradiation flow for thickness-direction in the PET film. The electric resistance $(R)$ of the PET film during soft X-ray irradiation can be obtained as approximately $1.0 \times 10^{8} \Omega$ from the slope of the approximated line of $I-V$ plot. Considering the SR beam size (estimated irradiation area of $3.7 \times 10^{-5} \mathrm{~cm}^{2}$ ), electric resistivity $(\rho)$ of the PET film can be estimated as $5.5 \times 10^{7} \Omega \mathrm{cm}$. In general, PET takes the higher $\rho$ of $10^{12}-10^{15} \Omega \mathrm{cm}$, which shows electric insulation. Hence, the lower $\rho$ during SR-beam irradiation clearly confirms that the electrical conduction path can be formed in the thickness-direction of the PET film.

In the $I-V$ measurements, $I$ and $V$ signals responded to the on/ off of SR-beam irradiation. In addition, no change of the $I$ and $V$ signal intensities can be observed during SR-beam irradiation for several tens of minutes at the fixed energy positions (250, 305,500 , and $541 \mathrm{eV}$ ). This confirms that the sample current
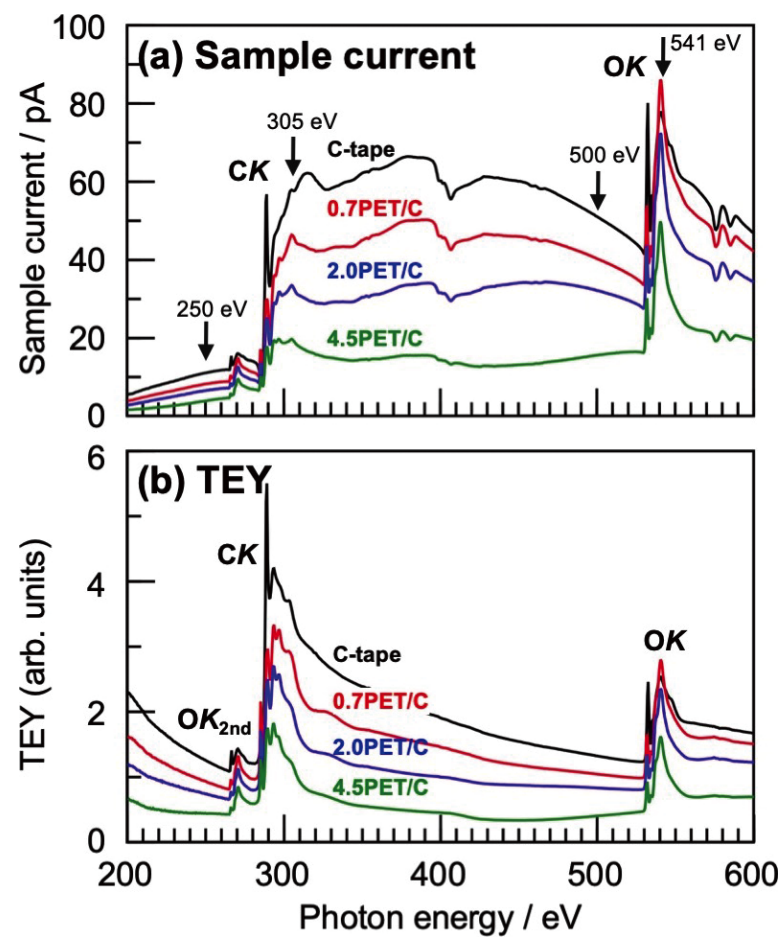

Fig. 8 Sample current (a) and TEY (b) spectra in the $200-600 \mathrm{eV}$ region of the PET/C-tape samples and a C-tape substrate. Thickness of the PET films are $0.7 \mu \mathrm{m}$ (denoted by 0.7PET), $2.0 \mu \mathrm{m}$ (2.0PET), and $4.5 \mu \mathrm{m}(4.5 \mathrm{PET})$.

surely flows in the thickness direction by SR-beam irradiation and that sample damage, including insulation breakdown by SRbeam irradiation, can be negligible.

\section{Thickness-dependent sample current measurements}

To estimate the attenuation length of sample current in PET films, thickness-dependent sample current measurements were performed by using the PET films with the thickness of 0.7, 2.0, and $4.5 \mu \mathrm{m}$, which are denoted by $0.7,2.0$, and 4.5PET, respectively. Figure 8 shows the sample current (a) and TEY (b) spectra in the $200-600 \mathrm{eV}$ region of the PET/C-tape samples with a C-tape substrate. It is clearly observed that the sample current and TEY decreased as the PET thickness increased. However, sample current can be sufficiently detected in several tens of $\mathrm{pA}$, and absorption edge profiles in $\mathrm{C} K$ and $\mathrm{O} K$ regions can be clearly obtained even in the thickest 4.5PET film. Figure 9 shows the sample current as the function of PET film thickness, which were measured at $250 \mathrm{eV}(\mathrm{a}), 305 \mathrm{eV}(\mathrm{b})$, $500 \mathrm{eV}(\mathrm{c})$, and $541 \mathrm{eV}(\mathrm{d})$ denoted by arrows in Fig. 8(a). These thickness-dependent sample currents can be well approximated with the function of $f(x)=A e^{-\mathrm{Bx}}$. This suggests that sample current intensity can be considered with the Lambert-Beer law, which is similar to the transmission of X-rays in matter. From the approximated curves, the attenuation length of the sample current can be expressed to be $1 / B$. The measured attenuation length can be obtained as $4-7 \mu \mathrm{m}$ as described in Figs. 9(a) 9(d). Such X-ray energy dependence of sample current attenuation length is reasonable, because attenuation length of $\mathrm{X}$-rays is also dependent on energy. Hence, the thicknessdependent measurements also confirm that the electrical conduction path can be formed in the thickness-direction of the PET film. 

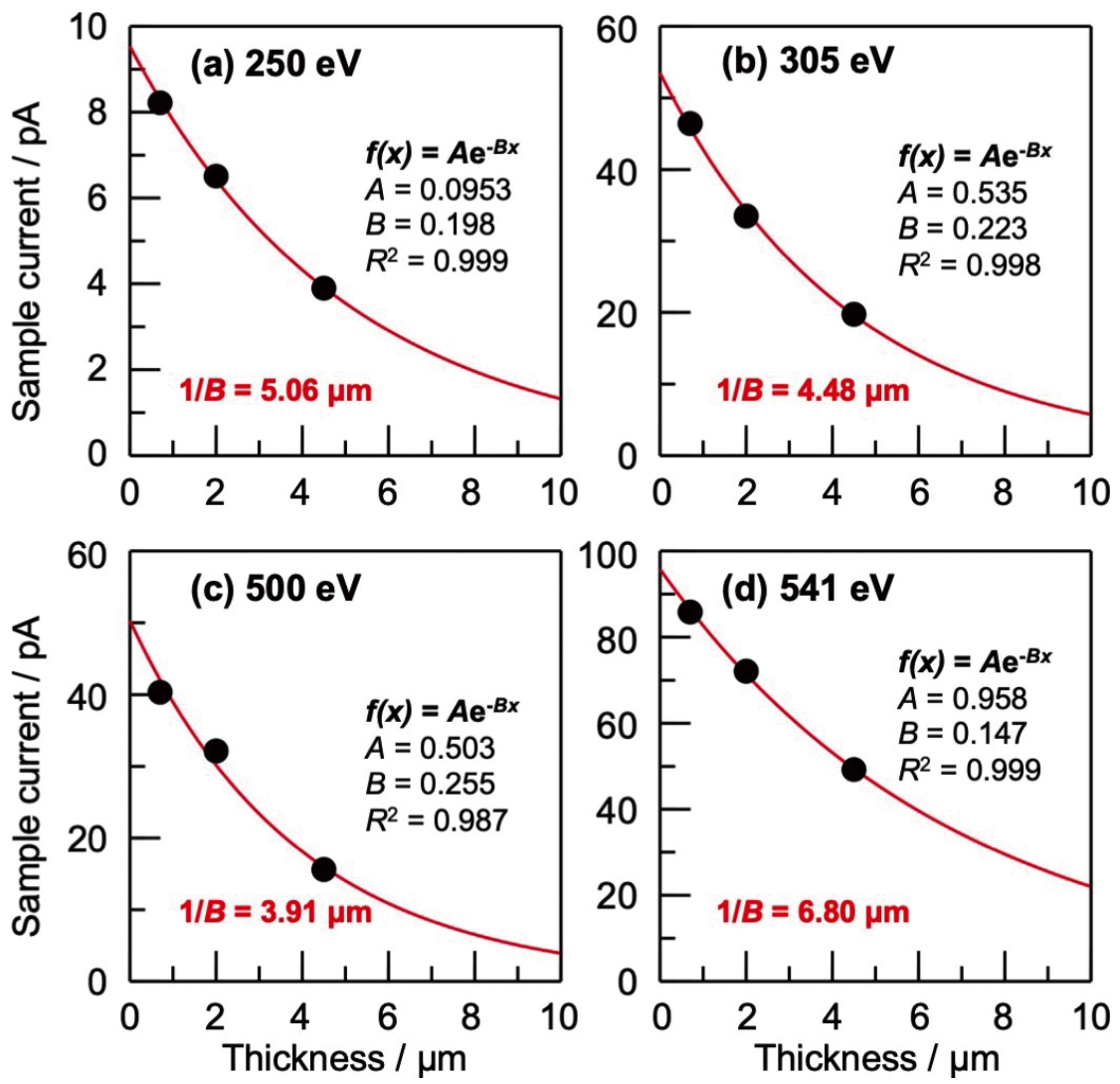

Fig. 9 Sample current as the function of PET film thickness measured at $250 \mathrm{eV}$ (a), $305 \mathrm{eV}$ (b), $500 \mathrm{eV}(\mathrm{c})$, and $541 \mathrm{eV}(\mathrm{d})$.

\section{Conclusions}

TEY-XAS measurements of the 0.7- $\mu$ m-thick PET/conductivesubstrates $(\mathrm{Au}, \mathrm{C}, \mathrm{Cu})$ provided XAS profile of PET, which were independent of the substrates. XAS measurements of selfstanding PET/Au and Au/PET films with the TEY and transmittance modes confirmed that the sample current of PET induced by soft X-ray irradiation flowed inside the PET film. $I-V$ measurements of PET films also confirmed that a conduction path should be formed in the thickness-direction of the PET film. Thickness-dependent sample current measurements provide data on the attenuation length of the sample current in PET films to be several $\mu \mathrm{m}$. From these measurements, it can be concluded that the sample current of insulating films placed on conductive substrates can be detected through the substrate. Such phenomena enable easy and simple TEY-XAS measurements for insulating samples.

Further measurements of thicker insulating samples (commercially available papers, clothes, and tapes) placed on conductive C-tape substrates have demonstrated that the measurable thickness is several tens of $\mu \mathrm{m} .{ }^{10}$ Therefore, the present method should be a simple and useful analytical tool for insulating thick film samples using the TEY-XAS.

\section{Acknowledgements}

The authors would like to express their gratitude to Dr. Takashi Shoji (Rigaku), Mrs. Koto Suganuma (Teijin), and Mr. Shuhei Yamamoto (Toray) for supplying the PET films. We would like to thank Mr. Takahito Ouchi (University of Hyogo) for his experimental support. This work was supported by a Grant-inAid from the Ministry of Education, Culture, Sports, Science and Technology of Japan under contract No. 18H02007.

\section{References}

1. J. Stöhr, C. Noguera, and T. Kendelewiez, Phys. Rev., 1984, B30, 5571.

2. J. Stöhr, "NEXAFS Spectroscopy", 1996, Springer-Verlag, $118-133$.

3. G. J. Baker, G. N. Greaves, M. Surman, M. Oversluisen, Nucl. Instrum. Methods in Phys. Res., 1995, B97, 375.

4. H. M. Wang and G. S. Henderson, J. Non-Cryst. Solids, 2008, 354, 863.

5. T. Ohkochi, M. Kotsugi, K. Yamada, K. Kawano, K. Horiba, F. Kitajima, M. Oura, S. Shiraki, T. Hitosugi, M. Oshima, T. Ono, T. Kinoshita, T. Muroa, and Y. Watanabe, $J$. Synchrotron Rad., 2013, 20, 620.

6. Y. Muramatsu and E. M. Gullikson, Adv. X-Ray Chem. Anal., Japan, 2017, 48, 317.

7. J. H. Underwood, E. M. Gullikson, M. Koike, P. J. Batson, P. E. Denham, K. D. Franck, R. E. Tackaberry, and W. F. Steele, Rev. Sci. Instrum., 1996, 67, 3372.

8. Y. Muramatsu, Y. Matsumoto, and E. M. Gullikson, Vac. Surf. Sci., 2020, 63, 470.

9. Y. Muramatsu and E. M. Gullikson, Adv. X-Ray Chem. Anal., Japan, 2010, 41, 127.

10. Y. Muramatsu, Y. Tani, Y. Tobita, S. Hamanaka, and E. M. Gullikson, Adv. X-Ray Chem. Anal., Japan, 2018, 49, 219. 